\title{
LA 'RESTITUCIÓN DE LA NIÑEZ’ COMO FORMA DE INCLUSIÓN SOCIAL EN UN CENTRO DE DÍA PARA "CHICOS DE LA CALLE” EN BUENOS AIRES ${ }^{1}$
}

\section{'CHILDHOOD RESTITUTION' AS A WAY OF SOCIAL INCLUSION, IN A DAY CENTER FOR "STREET CHILDREN" IN BUENOS AIRES}

\section{María Florencia Gentile*}

\author{
RESUMEN
}

¿Qué ocurre cuando los programas sociales "para niños" se dirigen a una población cuyas experiencias no coinciden con aquellas atribuidas a la noción hegemónica de infancia? En este artículo se analizará, a través del análisis de entrevistas y observación participante, los sentidos puestos en juego por los actores que interactúan en un programa social para niños y jóvenes "de la calle". Se verá cómo la concepción de niñez deja de ser descriptiva de la población destinataria, para convertirse en una noción moral y por ende, condición (no explicitada) de acceso y permanencia a la asistencia.

PALABRAS CLAVE: ARGENTINA * INFANCIA * NIÑOS DE LA CALLE * POLÍTICA SOCIAL * BIENESTAR DE LA INFANCIA* SOCIOLOGÍA DE LA INFANCIA * ENFOQUE RELACIONAL

\section{ABSTRACT}

What happens when social programs "for children" are directed to a population whose experiences do not match those attributed to the hegemonic notion of childhood? This article examines, through an analysis of interviews and participant observation, the senses put in action by the social actors that interact in a social

1 Este artículo es parte de los resultados parciales de la investigación doctoral en curso "Los 'chicos de la calle' y las nuevas propuestas para su inclusión social: interacciones, articulaciones y conflictos". Esta investigación cuenta con el financiamiento del Consejo Nacional de Investigaciones Científicas y Tecnológicas y de la Universidad Nacional de General Sarmiento. El trabajo de campo se desarrolló en dos etapas. Una primera, entre diciembre de 2004 y junio de 2006, que dio lugar a una Tesis para la obtención de un Máster en Sociología en la École des Hautes Études en Sciences Sociales de París (EHESS-París), en octubre de 2006. Y una segunda etapa, financiada con una beca del CONICET, en el marco del Doctorado en Ciencias Sociales de la Universidad de Buenos Aires.

Área de Sociología del Instituto de Ciencias de la Universidad Nacional de General Sarmiento (UNGS), Argentina. mflorgentile@yahoo.com 
program for "street children". It exposes how the concept of childhood is no longer descriptive of the target population and becomes a moral concept and therefore, a pre-condition (not explicit) for being admitted to and attended by the program.

KEYWORDS: ARGENTINA * CHILDHOOD * STREET CHILDREN * SOCIAL POLITICY * CHILD WELFARE * CHILD SOCIOLOGY * RELATIONAL APPROACH

\section{INTRODUCCIÓN. LOS “NIÑOS, NIÑAS Y ADOLESCENTES QUE VAN AL CENTRO DE DÍA" DESDE UNA PERSPECTIVA RELACIONAL}

Bryan y José, como lo hacen regularmente varias veces por semana, tocan el timbre del centro de día para chicos de la calle y esperan en la vereda que los coordinadores les abran la puerta para entrar. Son las 10:30 de la mañana y saben que están llegando más tarde de lo estipulado como horario de acceso (las 10 hs.). "Lo que pasa es que se nos rompió el carro", explica José cuando Martín (el coordinador) les recuerda el horario y luego acepta dejarlos pasar dando cuenta de una relación ya establecida ("solo porque son ustedes"). Pero en el momento en que Bryan está por cruzar la puerta, Martín se interpone y se produce el siguiente diálogo, en un tono de broma pero que al mismo tiempo deja traslucir cierta tensión creciente:

Martín - [entre desconfiado y jocoso] Che, Bryan ¿vos cuántos años tenés? Porque me parece que ya estás más grande...

Bryan - Tengo 17... [y desafiante, agrega] ¿No me creés? ¡Te muestro los documentos! ahí...?

Martín - [provocativo] ¿Los tenés

Bryan - ¡Claro! [y hace un gesto como si fuera a sacar algo del bolsillo trasero de su pantalón... pero no llega a sacarlo).

Martín - (cambia el tono, como dando a entender que ambos sabían que se trataba de un chiste) No, dale, entrá, está bien. ¡Te creo...! ${ }^{2}$

Sabemos, mediante Goffman que, una broma es una cosa muy seria en términos de interacción social. Una "simulación de discusión” en tono jocoso, como en este caso, es

2 Registro de campo. Fecha: 15-01-2005. un tipo de diálogo de "doble sentido" y puede poner de manifiesto un cierto desacuerdo con el consenso de trabajo acordado entre los equipos y necesario para toda interacción, pero manifestado de una manera ambigua o difusa, de manera tal, de no poner en riesgo el efectivo desarrollo de la actuación (1997: 205-215).

Durante mi estadía ${ }^{3}$ en el centro de día se presenció cotidianamente situaciones como la de Martín y Bryan, en las que se ponen en juego, se negocian y disputan (aún, como en este caso, de manera indirecta o "en broma") quiénes van a acceder a los recursos y servicios brindados y quiénes no. Es decir, los criterios para el acceso a la asistencia y en definitiva, la definición misma de la población que la institución se propone atender (los "niños/as en situación de calle"). Uno de estos criterios tiene que ver con la edad.

En este artículo (así como también en la investigación que le da origen) interesa hacer foco en estas prácticas e interacciones cotidianas entre los trabajadores del centro de día y quienes allí asisten. Ya que se adopta una perspectiva teórica y metodológica relacional que lleva a entender que en las negociaciones, acuerdos de trabajo (Goffman, 1997) y conflictos cotidianos, se redefinen $y$ ponen en juego los sentidos e implicancias de las intervenciones institucionales $y$ hasta la propia definición de

3 El trabajo de campo en el centro de día supuso dos etapas: una primera entre 2004-2006 y una vuelta al campo durante 2007-2008. La estrategia teórico-metodológica adoptada para el desarrollo de esta investigación es la de una mirada etnográfica (Beaud, Weber, 1997) que permita realizar un estudio comparativo entre dos programas del Área Metropolitana de Buenos Aires que trabajan desde la perspectiva de la "Protección Integral de Derechos": el centro de día que aquí se presenta, programa estatal con una retórica de la inclusión basada en la "ciudadanía" y otro no gubernamental, centrado en la idea de "transformación política". 
la población objetivo (los "chicos de la calle"), más allá de lo previsto formalmente por los programas. Esta perspectiva es tributaria de los aportes de Georg Simmel (2005), al estudio de la pobreza quien se aleja de los supuestos sustancialistas para establecer quiénes son "los pobres", para preguntarse por las respuestas sociales (formales-institucionales e informales) en tanto, parte de los mecanismos de construcción social para definir aquellas poblaciones que en cada momento histórico se considera, deben ser asistidas socialmente. En su obra Los pobres, Simmel sienta las bases de esta perspectiva:

Los pobres [y nosotros podríamos especificar: los chicos de la calle], en tanto categoría social, no son aquellos que sufren faltas o privaciones específicas, sino aquellos que reciben asistencia o deberían recibirla según las normas sociales. En consecuencia, la pobreza no puede, en este sentido, ser definida como un estado cuantitativo en sí mismo, sino que sólo puede ser definida en relación a la reacción social que aparece como respuesta a una situación específica [...] (2005: 96-97).

A su vez, sostenemos que esta construcción no es unidireccional, sino que estas categorías sociales se van definiendo en el curso mismo de las interacciones cotidianas, en un proceso en el que participan activamente tanto quienes trabajan en una institución, como los propios usuarios y/o destinatarios de esos dispositivos (Pichon, 2005). Aunque esta participación no sea simétrica e implique, como se verá, jerarquías y distintas posiciones de poder. En congruencia con la perspectiva teórica adoptada, se entiende que la categoría "chicos de la calle" no define a priori una situación sobre la que las instituciones actuarían, sino que es el resultado del proceso de estas interacciones cotidianas.

Volviendo entonces al centro de día, se trata de una institución para niños, niñas $y$ adolescentes en situación de calle del Gobierno de la Ciudad de Buenos Aires. De manera formal, establece su población objetivo como "niños, niñas y adolescentes de 8 a 18 años de edad $^{4}$ que viven, deambulan y/o trabajan en las calles de la Ciudad de Buenos Aires" (Proyecto Institucional, 2003). El objetivo del programa es la "atención, contención, diagnóstico, acompañamiento y derivación" de estos "niños, niñas $y$ adolescentes en situación de calle". Abrió sus puertas a mediados de 1992 inspirado en las definiciones de la Convención Internacional sobre los Derechos del Niño y trabaja bajo la forma de concurrencia directa $y$ voluntaria de los niños y jóvenes ${ }^{5}$. Sus objetivos son los de brindar atención a esta población desde la asistencia a sus necesidades diarias, actividades educativas y de recreación, el acompañamiento en situaciones que demanden contacto con otras instituciones, hasta la elaboración de estrategias para la salida paulatina de la situación de calle. En términos edilicios, se trata de un galpón ubicado en la zona sur de la Ciudad de Buenos Aires, relativamente cerca de la zona céntrica de la ciudad (por cuyas calles circulan $y$ permanecen los niños y jóvenes que se acercan a la institución) y en un lugar con buen acceso (múltiples líneas de buses, cercanía de una de las terminales de trenes más importantes) lo cual resulta fundamental para el acercamiento cotidiano.

Más allá de las definiciones formales, una de las cosas que llamó la atención durante el trabajo de campo fue la presencia cotidiana en el centro de día de niños pequeños, menores de 8 años y en mayor medida, de jóvenes mayores de 18. De hecho, en varias oportunidades se realizaron entrevistas dentro de la institución a "chicos" que tenían 20 o 22 años y que muchas veces se podían ver jugando al metegol, al fútbol, dibujando en algún taller o cantando junto con otros chicos de menor edad. El procesamiento estadístico de los legajos con

4 En línea con la definición presente en la Convención Internacional sobre los Derechos del Niño, que establece que "se entiende por niño todo ser humano menor de dieciocho años de edad" (CIDN, 1989).

5 Y no, como la mayor parte de las instituciones que trabajan con niños que son llevados allí por otros adultos (padres, jueces, profesionales de otras instituciones, etc.). 
datos sobre los asistentes permitió confirmar y dimensionar esta observación: si bien el grueso de la población asistente tiene entre 13 y 16 años, existe un 5,4\% de los asistentes que tiene menos de 8 años (algunos de los cuales son bebés de las adolescentes que asisten a la institución) y un $10 \%$ que supera el límite estipulado de edad, con 19 años o más (llegando hasta los 24 años $)^{6}$. De la misma manera, también se observo cómo se decidía a veces que algunos "niños/as o adolescentes" que se encontraban en el rango de edad definido como población objetivo, no entrarían al centro de día por uno o dos días, generalmente por haber protagonizado situaciones conflictivas en visitas anteriores.

Explícitamente, la edad de los asistentes aparece como un criterio para definir a los "niños y adolescentes" a los que está destinado el dispositivo (aquellos que tienen entre 8 y 18 años) y este criterio es conocido por quienes allí concurren. No es extraño entonces, que (como lo hace Bryan) estos "chicos" respondan con cierta ambigüedad al ser indagados sobre su edad o directamente respondan tener una edad un día y otra al día siguiente, de acuerdo a la situación, frente a quién se realice la presentación y también a la utilidad que pueda brindar en cada ocasión el hecho de tener 14, 16 o 21 (por ejemplo, el acceso a ciertos programas sociales, becas, el propio acceso al centro de día, evitar se llevados por la policía, etc.) ${ }^{7}$.

6 Sistematización de los datos sobre el total de asistentes de 2003. La distribución etaria de los asistentes se mantiene constante a lo largo de los años. Para una caracterización sociodemográfica de la población asistente al centro de día. Ver Gentile, 2006 y Pojomovsky, colab. Cillis, Gentile, 2008 .

El hecho de mentir sobre la edad (y particularmente, sostener que es menor de 18 años, aún cuando ya se superó esa edad) es una práctica recurrente señalada en las investigaciones sobre esta población. Por ejemplo, esta misma observación es realizada en un trabajo etnográfico sobre chicos de la calle en Sao Paulo, Brasil. Allí, la autora interpreta que esta "mentira" tiene que ver con que "la mayoría de edad para los chicos $-y$ especialmente, para aquellos que están en la calle desde temprano- no implica solamente un cambio de edad o de identidad, sino también un cambio de relaciones, ya que ser mayor significa
Los coordinadores, por supuesto, saben que esto ocurre; sin embargo, este no parecería ser un criterio taxativo o, en todo caso, el único criterio puesto en práctica, para decidir la asignación de la asistencia a ciertas personas (y a otras no). Cualquier mirada distraída podría imputar rápidamente una "contradicción" entre lo que la institución proclama formalmente y lo que realiza de manera efectiva ( $y$ abogar por su "coherencia"), o resaltar la "tolerancia" de los coordinadores. Sin embargo, vale la pena interrogar esta aparente "contradicción", ya que ella puede servir para comprender y recrear las lógicas que ponen en práctica los propios actores, en las que residen los criterios efectivos que se ponen en juego en las interacciones cotidianas locales para dirimir y delimitar la población real que el programa va a asistir y sus límites. Y esto, porque retomando lo señalado por Gerard Mauger, al analizar el caso francés, "es sólo a este nivel que es posible tener medida de los 'efectos' de tales dispositivos estatales, que con frecuencia difieren de aquellos que son explícitamente propuestos y esperados" (2001: 4).

La hipótesis que interesa abordar en este artículo es que en los criterios prácticos para tal definición ( $y$ en las negociaciones $y$ disputas alrededor de ellos), lo que está en juego es la propia noción de niñez. Y por lo tanto, se propone recrear las maneras en que quienes trabajan en el centro de día y también quienes allí se acercan para ser atendidos, definen en interacciones cotidianas quiénes son niños ("en situación de calle"), o no lo son.

tener una relación diferente con la policía, con las ONGS, con el tráfico y con los otros chicos de la calle" (Frajtag Sauma, 2006: 55). 


\section{LA NOCIÓN DE NIÑEZ EN EL CENTRO} DE DÍA

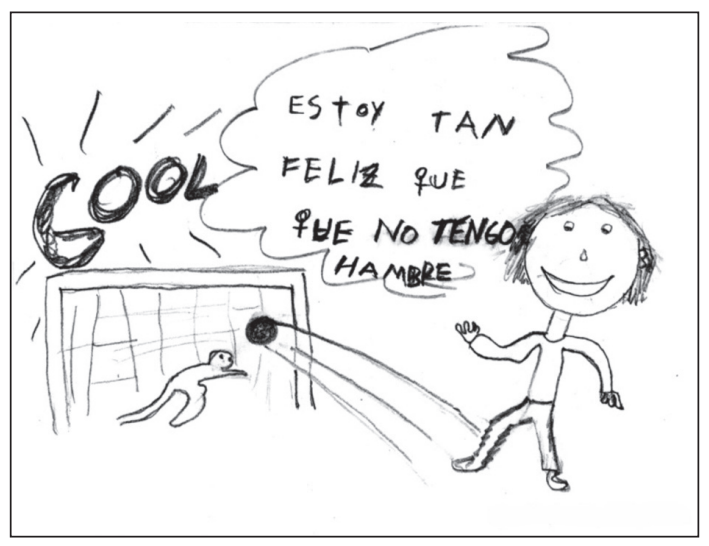

Roberto, 14 años ${ }^{8}$

El dibujo de Roberto, realizado en un taller y publicado en una revista de la institución, logra sintetizar de manera gráfica la manera en que desde la institución se comprenden a los "niños, niñas y adolescentes" que allí participan y por ende, el propio rol de la institución.

En efecto, desde el centro de día se entiende que los niños y adolescentes que se acercan cotidianamente, debido a las condiciones sociales en las que viven (pobreza, maltrato, tratamiento institucional inadecuado), fueron impedidos de vivir estos años de sus vidas acorde a las experiencias identificadas como propias de la niñez en nuestras sociedades. Esta interpretación es posible porque la edad no es el único criterio para diferenciar quiénes son niños y quiénes no lo son en nuestras sociedades. La norma hegemónica acerca de lo que implica ser un niño remite a su vez a una serie de experiencias, que son producto de los sentidos asociados culturalmente a la infancia. Las experiencias que no correspondan con ella, son interpretadas entonces como desvíos, como estados "fuera de la niñez". En nuestras sociedades latinoamericanas, la idea de niñez que opera como norma (y que las instituciones reproducen) se basa en las vivencias de las

$8 \quad$ Publicado en la revista Chicos de la calle. Editada por el centro de día, 1998. clases medias urbanas, no obstante, las experiencias de otros grupos sociales y/o culturales que difieran con ellas son concebidas como desvíos (Fonseca, 1998: 27-43) ${ }^{9}$. A estos sectores medios pertenecen, a su vez, los educadores que trabajan con los asistentes en el centro de día.

De manera sintética, podríamos identificar que la manera hegemónica en que se concibe la niñez en nuestras sociedades, contiene tres elementos que la distinguen (al mismo tiempo que definen, ya que estas categorías no pueden sino pensarse en forma relacional) de lo propio de la adultez. Por un lado, como muestra el historiador Philippe Ariès, la concepción de una diferencia radical con el mundo adulto, que construye una separación tajante de espacios, prácticas, relación con los objetos, comportamientos, sentimientos y hasta "modos de ser" concebidos como propios de lo adulto y de lo infantil (1988). Por otro lado, una jerarquía entre lo adulto y lo infantil, derivada de la idea de niño como ser inacabado y por ende, frágil, vulnerable $y$ dependiente. Y por último, la particularidad del binomio infancia-adultez, que si bien comparte con otros pares de categorías de opuestos, la diferenciación y la jerarquización (por ejemplo, en la construcción de lo masculino $y$ lo femenino) en el caso de la infancia, esta supone un estado capaz de transformarse, con el tiempo, en su opuesto, la adultez. Se desarrolla entonces una idea de pasaje necesario, de preparación progresiva para la vida adulta a través de fases de desarrollo (en consonancia, como muestra Fonseca, 1998, con la experiencia de las clases medias urbanas).

La experiencia de la vida en las calles de los chicos del centro de día, pone en tensión cada uno de esos elementos y por lo tanto, pone de manifiesto, a la vez que cuestiona, el ordenamiento moral en el que se basan estas construcciones sociales. Tanto desde la indiferenciación del espacio de la calle, en el que conviven y se mezclan adultos y niños; desde la adopción de conductas necesarias para la sociabilidad en ese medio social e identificadas culturalmente

$\overline{9} \quad$ Los trabajos etnográficos de Claudia Fonseca contraponen esta noción hegemónica con la noción de infancia en los sectores populares de Porto Alegre, Brasil. Cfr. (Fonseca, 1998: 27-43). 
como conductas "de adultos"; desde la autonomía e inversión de jerarquías que supone un niño viviendo autónomamente de un adulto; características todas que cuestionan la idea de pasaje progresivo $y$ suelen ser interpretadas como un pasaje compulsivo y temprano a la adultez $^{10}$.

Desde la visión de la institución, entonces, la situación de exclusión social en la que se encuentran estos chicos se traduce específicamente en la exclusión de la propia experiencia de la infancia. Se trata de niños y jóvenes "con la infancia vulnerada".

El recurso discursivo de la Convención Internacional de los Derechos del Niño (CIDN), al que se apela como marco de trabajo cotidiano, se convierte así en un principio de inteligibilidad de la situación de estos niños y jóvenes, $y$ en un principio de legitimación del accionar de la institución: estos han sufrido, por ser "menores" (sin capacidad para ejercer derechos, noción propia de la legislación vigente hasta 2005 en Argentina), por ser pobres (excluidos socialmente) y por estar en la calle (sin la protección de adultos y a merced de las "instituciones represivas", como la policía y los institutos de encierro), una sistemática e histórica "violación de sus derechos". La "misión" del centro de día se plantea, dentro de este universo de sentido, como una intervención que los "restituya como sujetos de derechos". Pero en el trabajo cotidiano, se opera una "traducción" específica de este postulado legal: de la defensa de

10 Aparece así la figura del "niño trabajador" o del "niño-adulto". Como ejemplo de esta figura se puede mencionar, el trabajo de Mariela Macri: El trabajo infantil no es juego, en donde da cuenta de esta concepción de la "infancia pobre" como "infancia trabajadora". Al esbozar la historia del trabajo infantil, Macri describe que "las condiciones de vida de los sectores populares, ya sean campesinos u obreros, obligaron a que sus hijos realizaran prácticas de trabajo en condiciones infrahumanas $y$ de poco valor formativo. La temprana inserción de los niños en la vida laboral favoreció la cristalización de una imagen de 'niñoadulto' que comenzó a ser exclusiva para los sectores de la sociedad más empobrecidos. Para ellos se perdió el carácter lúdico de la vida cotidiana y su exclusión de la escolaridad reveló la negación de su condición de niños" (Macri y otros, 2005: 21). "los derechos del niño" (en la que el estatus de niño está dado y el problema son sus derechos vulnerados), se pasa a la defensa del "derecho a ser niño", ya que se interpreta que es el propio hecho de constituirse como tal lo que está vulnerado.

Las palabras de este educador en una entrevista, explican claramente esta concepción. Ante la pregunta de ¿̇por qué pensaba él que los chicos se acercan voluntariamente al centro de día?, respondió:

Yo creo que los pibes vienen a buscar... eh... vienen a buscar lo que mejor da el centro de día, digamos, que es un espacio donde pueden ser un poco más "chicos” que en sus otros espacios. Y es el "ser chico" en toda su amplitud: el chico enojado, el chico enojado con un adulto, el chico que juega, el chico que abraza. Pero como chico, digamos. Creo que son pibes que no pueden, que les cuesta esto en otros ámbitos. Por lo menos lo pueden hacer pero con mucho riesgo, digamos. Me parece que el jugar en la calle es arriesgado, el jugar seguramente en el barrio no es tan fácil. Y el [centro de día] es un espacio de juego... de ser chico ¿no? (...) Y creo que... vienen a buscar a un adulto... que sea interesante para permitirle esto. Y me parece que el equipo del aquí es muy bueno realmente, y que permite esto para el pibe, ¿no? (... $)^{11}$.

Otras investigaciones han identificado una noción similar, por ejemplo, la de "infancia interrumpida", en el caso de los niños internados en el Hospital pediátrico de Pequeño Príncipe, Brasil (Fioravanti, 2006). Ese trabajo muestra que allí, los niños son percibidos por los voluntarios que trabajan con ellos como crianças doentes (niños enfermos), a quienes la fatalidad del padecimiento de una enfermedad los llevó a ver "interrumpida" su infancia. En ese caso, los voluntarios distinguen la situación de estos niños de cualquier otro "en situación de riesgo", por el hecho de que, al haber una enfermedad, "nadie es responsable"

11 Entrevista a educador. Fecha: 02-02-2005. 
de la situación que viven (ni sus familias, ni ellos mismos). La visión de quienes trabajan en el centro de día que se estudia en el artículo, difiere en este punto, ya que si bien coinciden en comprender que estos niños $y / 0$ adolescentes han visto impedida la posibilidad de desarrollar su infancia (en relación a lo estipulado socialmente como tal), muchas veces se asocia esta situación con la existencia de adultos, que por distintos $y$ a veces hasta justificados motivos, "no han cuidado/protegido" a estos niños para que puedan "ejercer" su niñez. Estos adultos pueden tratarse en algunos casos de los miembros de la familia de esos chicos, o de otros adultos en la calle, pero también desde el centro de día se percibe así a las instituciones estatales de tradición tutelar ${ }^{12}$, que históricamente utilizaron la judicialización de las problemáticas sociales y el encierro en "Institutos de menores" como formas privilegiadas de tratamiento a la cuestión social de la infancia. Estos adultos serían responsables, en tanto, no han "cuidado y protegido", y por lo tanto, no permitieron que estos chicos "puedan ser niños".

De esta manera, más allá de lo proclamado explícitamente, las actividades desarrolladas desde el centro de día ( $y$ su "tarea de reparación", en palabras de Goffman, 1972) cobran otro sentido: sí su población consiste en "chicos con la infancia vulnerada", la reparación social y la reinclusión de estos chicos "excluidos" que promueve la institución, así como, la "restitución de derechos" que se propone, es concebida en términos de la restitución de la niñez. Si en parte esto se entiende como producto de la relación con adultos que no los han protegido, en este centro de día se les cuida, se les acompaña, se les protege, es decir, se desempeña un rol de adulto que les brinde la posibilidad de ser niños, a pesar de sus historias y de las condiciones en las que viven.

Esta manera de concebir la acción institucional tiene su historia. El surgimiento $y$ consolidación de esta institución gubernamental se produjo en un contexto de vigencia de las ideas neoliberales que signaron las políticas públicas (entre ellas, las sociales) en la década

12 La tríada policía-juez de menores-institutos de menores. del 90 en la Argentina. Al mismo tiempo, el centro de día surgió como una propuesta alternativa de tratamiento de la cuestión social de la infancia, en una época en la que aún estaban vigentes normativas opuestas al "Paradigma de la Protección Integral” (propio de la CIDN) y por lo tanto, ocupó históricamente una posición subordinada dentro del campo de la asistencia a la infancia pobre. Ambas situaciones llevaron a que la institución haya tenido que enfrentarse desde sus inicios a una fuertísima carencia de recursos disponibles (de personal asignado al programa, de infraestructura, de material de trabajo, etc.).

Es así como la institución fue concebida como una pequeña parte de un gran proyecto de asistencia a los "chicos de la calle" (que incluía operadores en calle, múltiples centros de día y pequeños hogares a los que se derivarían los niños como propuesta alternativa a la situación de calle) y finalmente, fue la única institución que se inauguró. Así, los objetivos de la institución, la concepción de su tarea y su misión fueron cambiando a medida $y$ en función de la disponibilidad de recursos con los cuales trabajar, estos mismos a su vez producto de la lucha dentro del campo, de la posición ocupada por el centro de día y también de la constitución del problema de los "chicos de la calle" como una prioridad social.

Con el tiempo, al no tener disponibles otros soportes materiales e institucionales para ofrecer una alternativa efectiva a la situación de calle a niños y jóvenes, que permita modificar sus condiciones sociales, los objetivos institucionales fueron cambiando en la práctica. El objetivo de lograr que estos niños y jóvenes abandonen la situación de calle fue dando progresivamente prioridad a la experiencia que se produce con ellos dentro de la institución (aunque esta siga concibiéndose como parte de un proceso de salida progresiva de la calle). Así fue delineándose de a poco la "misión" actual del centro de día; por un lado, el posibilitar que estos chicos sean, al menos por un rato (en las horas que pasan dentro del centro de día $y$ solo en aquellos días que concurren), niños como los otros. Y por otro lado, la idea de que esta "transformación" que se opera dentro del 
centro de día, pueda reparar aquello que estos chicos tienen vulnerado.

\section{LA "RESTITUCIÓN DE LA NIÑEZ" COMO FORMA DE INCLUSIÓN SOCIAL}

¿Cómo se intenta llevar a cabo desde el centro de día, entonces, este trabajo de reparación que permita restituir la "infancia vulnerada" de estos "niños y adolescentes en situación de calle"? El trabajo de rehabilitación que se propone en el centro de día es producto del entrelazamiento de dos tradiciones históricas de la asistencia a estas poblaciones; la tradición estatal de promoción de la ciudadanía y la tradición de las organizaciones sociales y humanitarias de la sociedad civil que en América Latina tuvieron una gran importancia en el trabajo con la población de "chicos en situación de calle"13. Por un lado, la "restitución de la niñez" se presenta a la manera de las organizaciones humanitarias, que conciben la reinserción social de las personas excluidas, a través del "trato humano" y de considerarlos como "seres humanos dignos" (Parizot, 2003), discutiendo $y$ denunciando los tratamientos sociales que estigmatizan y al hacerlo, deshumanizan a estos chicos. Se trata entonces de "tratarlos como niños", es decir, como se trata a otros niños, brindándoles cuidado, afecto y protección. Pero al mismo tiempo, en tanto institución gubernamental, se piensa la "restitución de la niñez" como una forma de acceso a la ciudadanía; la posibilidad de que estos niños sean reconocidos/tratados de igual manera que los otros niños, con los mismos derechos que otros niños tienen, independientemente de su situación económica y social, en tanto miembros de la comunidad política (niños-ciudadanos) ${ }^{14}$.

13 Esta distinción se basa en la identificación de dos "universos simbólicos" que históricamente han orientado las acciones de asistencia a las poblaciones desvalidas y que Isabelle Parizot identifica al estudiar el caso francés (Cfr. Parizot, 2003).

Desde una definición clásica del concepto de ciudadanía (Marshall, Bottomore, 1998), que refiere a la posesión de derechos formales habilitantes de un tratamiento igualitario de los ciudadanos, en tanto miembros de una sociedad, al mismo tiempo que ubica al Estado como garante de esos dere-
En este sentido, se denuncia el tratamiento institucional que históricamente recibió esta población, que cataloga a estos niños como "menores" y los deriva a un circuito institucional (policía-juzgados-institutos) que los encierra y diferencia del resto de los niños (y sus instituciones: la escuela). Asimismo, la concurrencia voluntaria al centro de día (característica de la metodología de trabajo) es entendida también como el respeto a la voluntad de estos niños y el derecho de ejercerlo, más allá de los condicionamientos sociales en los que se encuentran. El centro de día se propone así restituir a los niños en tanto "sujetos de derechos", es decir, rehabilitar una figura particular de niño: la de "niño-ciudadano".

La atención efectuada a estos chicos va a intentar, que al menos dentro del tiempo y el espacio controlados por la institución, quienes allí se acerquen "puedan ser niños" (y más particularmente: niños-ciudadanos). Este sentido de la "rehabilitación de la infancia" se realiza a través de diferentes prácticas: la escenificación del espacio de los intercambios cotidianos, las actividades que se proponen en él y el tipo de trato brindado a estos chicos. Estos elementos se ilustran en el siguiente extracto de las notas de campo ${ }^{15}$ :

El centro de día es una especie de galpón que cuenta con un gran salón central, lugar principal por donde circulan quienes allí asisten. En él pueden observarse grandes mesas rectangulares y sillas, utilizadas para servir el desayuno y el almuerzo, y también para la realización de los talleres de expresión y recreativos. Muchas veces suelen encontrarse sobre ellas juegos de mesa para que los chicos jueguen entre sí o con los coordinadores. En el otro costado del salón, el espacio vacío es sólo entrecortado por la presencia de dos "metegoles", en el cual es corriente ver jugar a los chicos entre sí o

chos. Para una interesante revisión de los autores que trabajaron la idea de ciudadanía vinculada a la infancia, ver Litichever, 2009.

Registro de campo. Fecha: 17-01-2005. 
con algunos coordinadores. Las paredes de la primer parte del salón están decoradas con lo producido en los talleres: dibujos, collages, manualidades $y$ hasta un mural pintado por uno de los asistentes. También hay carteles que anuncian las actividades de los talleres. La exposición de los dibujos y producciones da una idea de un espacio de recreación y de aprendizaje específico para niños, en los cuales los chicos tienen un lugar de "coproducción". Este es el lugar por el que circulan mayoritariamente los chicos que asisten, en donde pasan la mayor parte de su tiempo durante la jornada en el centro de día, sentados en las sillas o sobre las mesas, parados, charlando entre sí, corriendo, participando de algún taller, charlando con los coordinadores, jugando. Es el espacio de la interacción por excelencia, la escenificación del contacto diario entre los chicos y la institución se da en esta región.

Al fondo del galpón, como continuación del salón principal pero distinguiéndose en parte, se encuentra "la cancha", lugar donde se desarrollan actividades de despliegue físico, como jugar al fútbol, al básquet, correr. Allí también tienen lugar los talleres que requieren de tal despliegue, como el taller de circo (donde los chicos realizan actividades de acrobacia) y el de murga (donde los chicos tocan instrumentos, bailan y arman coreografías).

En este extracto de las notas de campo sobre la descripción del lugar, puede observarse el intento por recrear un espacio propio de niños $y$ diferenciado de los espacios de adultos, que pone en escena las actividades consideradas adecuadas y propias de la infancia (hacer dibujos, jugar, realizar actividades recreativas). La idea de poner a la vista lo que se realiza en los talleres da una idea de que quienes asisten "participan activamente" del centro de día, son en parte "coproductores", lo que remite a ciertos atributos propios de la noción de niñociudadano ${ }^{16}$.

16 En tanto manifiesto jurídico de la "ciudadanía infantil", la CIDN establece entre sus principios
Las distintas actividades que se realizan (y las que no) también dan cuenta de estas tradiciones de la asistencia, desde la cual se concibe la tarea de la reparación de la infancia. En el siguiente extracto de una entrevista con uno de los coordinadores, aparecen estas tradiciones nuevamente puestas en juego, en la justificación de por qué en el centro de día no se ofrecen talleres de oficios o capacitación laboral (como sí ocurre en otras instituciones que trabajan con esta población):

(...) es cierto que a mí no me gusta que un chico de catorce años haga un curso de un oficio "para pobres". Yo prefiero que ese chico aprenda a andar en zancos, y que sea un excelente músico. Que se encuentre cantando frente a cincuenta personas y sienta que fue uno de los mejores momentos de su vida. Y no que haga un curso para aprender a hacer trapos de piso con papel de diario. Ahí es mi opinión. (...)Ahora, también, hubo un chico que fue al taller de actuación que hicimos y ganó plata como actor. Otro chico hizo artesanías y recorrió el país con eso. En esos dos casos tenés una relación con lo laboral... Inclusive, desde lo laboral como a mí me interesa, porque cada uno pudo poner cosas de ellos en el laburo. No es que le enseñamos a hacer con papel de diario dos trapos de piso para vender por ahí. 0 panadería para ser un fucking gastronómico. No es eso. ¿Por qué no pensar en que terminen el secundario para entrar a la Universidad? Enseguida se piensa: "Son pobres, que aprendan un oficio". ¿Y tu nene, que tiene 16 años $y$ es un vago que mira todo el día la tele e Internet... qué oficio le estás enseñando? "Ah, no, bueno, a él, no..."17.

Aquí aparece la idea del "derecho a ser niño" (y a no realizar actividades "de adultos",

rectores el derecho de los niños a la participación, opinión y escucha sobre temas que tengan que ver con su interés (1989).

17 Entrevista a coordinador del programa. Fecha: 10-02-2005. 
como trabajar) y al mismo tiempo, la tradición ciudadana de tratar a estos chicos de la misma manera que se trata a otros niños de otros sectores sociales de nuestra sociedad (con las mismas aspiraciones y proyectos).

Las estrofas de la siguiente canción preparada por los educadores para estos niños $y$ jóvenes como regalo de Navidad, también dan cuenta del sentido que se otorga a la asistencia brindada y por lo tanto, de la manera en que se concibe a quienes allí se acercan:

En este día de hoy

Al CENTRO yo vengo a cantar

Para festejar este año

Hay cosas para recordar.

Yo sé que no todo fue bueno

Pero se pudo superar

Con la alegría de ustedes

El CENTRO volvió a funcionar.

(...)

A ti te quiero decir

Pasá feliz Navidad

Que el año nuevo ya llega

Y el CENTRO siempre estará.

Queremos agradecerles oh...oh...

Lo mucho que ustedes nos dan.

Estas puertas están abiertas

Vengan a jugar oh...oh...

Estas puertas están abiertas

Vengan a jugar oh...oh... 18

Si relacionamos esta canción con los elementos constitutivos de la niñez hegemónica que se presentaron anteriormente, vemos que en esta canción aparece la idea de que el centro de día es un lugar para cantar y para jugar (actividades identificadas como distintivas de la infancia), que allí los niños pueden ser/estar alegres, lo que remite a los atributos personales asignados socialmente al "ser" niño y la idea de que el centro de día, al igual que una familia, "siempre estará", asumiendo la figura del adul-

18 Material de campo recogido en ocasión de las fiestas de diciembre de 2004. to que garantiza y posibilita el ejercicio de la niñez. El énfasis en las "puertas abiertas" no es solo una metáfora de una permanente bienvenida (a modo de una familia), sino que remite también a la distinción con las instituciones de encierro o "de puertas cerradas" (ciertos hogares o institutos) y en este sentido, a la idea de respeto a los derechos de esos niños (niño ciudadano).

Otra de las prácticas a través de las cuales se busca la "restitución de la niñez", tiene que ver con el modo de relacionamiento propuesto. Una de las propuestas hace referencia a la exclusión del uso de la fuerza física como criterio válido de relación (propia del espacio de socialización de "la calle") ${ }^{19}$ dentro del espacio de la institución, que lleva a interpretar que quienes se comportan a través del uso de la fuerza física están actuando de manera "violenta" y por lo tanto, se los "saca" del centro de día (con riesgo de quedar excluídos del programa de asistencia si persisten en su actitud). Otra propuesta tiene que ver con aprender a expresarse a través de manifestaciones de afecto y cariño, que también aparecen como maneras de acercarse a las prácticas y "formas de ser" consideradas más adecuadas a la idea de niño. La importancia otorgada a la expresión a través del uso de "la palabra" (tanto por parte de los chicos como por parte de los educadores que "hablan" con ellos) ${ }^{20}$ da cuenta también del fomento de la adquisición de capacidades propias del ejercicio ciudadano (por ejemplo, expresar su parecer sobre las cuestiones públicas). Ambas expresiones llevan también a reem-

19 En la investigación se identificó que los niños y jóvenes dan cuenta de la fuerza física como un criterio válido para relacionarse en el espacio social que identifican como "la calle", tanto para relacionarse entre sí, como con adultos y con ciertas instituciones como la policía y los institutos de menores. Esta forma de relación implica un estado de alerta permanente y la demostración de habilidad en el manejo del capital físico (Mauger, 2006), así como el establecimiento de jerarquías determinadas entre quienes lo poseen ("se la bancan") y quienes no ("no tienen aguante”). Cfr. Gentile, 2009.

20 La idea de la importancia que cobra el uso de "la palabra" como propuesta resocializadora del centro de día, está más desarrollada en Gentile (2009). 
plazar los capitales $y$ habilidades que los niños $y$ jóvenes asistentes poseen y manejan con mayor destreza que los adultos que allí trabajan (como lo es la posesión y el uso de la fuerza física), por otros criterios que implican el uso de recursos (la palabra, una cierta expresividad afectuosacariñosa) en la que los educadores se sienten más diestros, y que no todos los niños y jóvenes asistentes poseen y manejan en igual medida. De esta manera, los niños y jóvenes del centro de día quedan re-ubicados en un lugar subordinado en la relación con los adultos que allí trabajan, reconstruyendo la relación asimétrica implícita en la noción de infancia.

\section{IV. ¿QUIÉN ENTRA Y QUIÉN NO? CUANDO “SER NIÑO” PASA DE SER UNA DESCRIPCIÓN DE LA POBLACIÓN A SER UNA CONTRAPARTIDA MORAL EXIGIDA}

Se ha visto cómo, ante el hecho de encontrarse con personas en las que la edad $y$ las experiencias de vida en la calle no concuerdan con lo esperado como propio de la niñez en nuestras sociedades, desde el centro de día se opera una suerte de "infantilización", en el sentido de realizar un tratamiento que los acerque a las experiencias $y$ vivencias consideradas propias de la infancia hegemónica. Al menos, durante el tiempo transcurrido dentro de la institución.

Sin embargo, si desde quienes allí trabajan se intenta otorgar a estos niños y jóvenes la "oportunidad" de expresar y desarrollar "su naturaleza de niños" (y que implica también la posibilidad de ejercer el rol de "adulto protector y comprensivo" por su parte), desde los niños y jóvenes que asisten, la propuesta no tiene nada de "natural" y por el contrario, contradice una parte importante de sus propias vivencias, recursos, habilidades $y$ sentidos adquiridos en el espacio social de la calle. Desempeñar el rol de niño propuesto por el centro de día supone el manejo de recursos $y$ habilidades que no todos tienen por igual y para algunos, puede incluso convertirse en algo extremadamente difícil de sostener. Los chicos que allí asisten saben que para acceder a los recursos y servicios pueden, pero a la vez, deben, "comportarse como niños”. Y entonces, los niños y jóvenes, en tanto actores sociales plenos (Cohn, 2005), aceptan, negocian y/o disputan el desempeño del rol propuesto por la institución.

La descripción de la situación del almuerzo que se presenta en el siguiente recuadro, basada en extractos del cuaderno de campo, pone de manifiesto cómo en la interacción cotidiana entre los trabajadores de la institución y los niños y jóvenes que asisten, se opera, no exenta de conflictos y tensiones entre unos y otros, la construcción social de la niñez (y la adultez) dentro de este centro de día.

\section{TENSIONES Y CONFLICTOS COTIDIANOS: EL MOMENTO DEL ALMUERZO}

La comida que se sirve cotidianamente en el centro de día consiste en un plato principal y un postre. Los chicos reciben su plato $y$ suelen sentarse en las mesas agrupándose espontáneamente en grupos de pertenencia. La distribución de los educadores, si bien no está prefijada, intenta garantizar que haya al menos un adulto por mesa. Esta presencia en las mesas genera una situación ambivalente, que escenifica una tensión típica de las interacciones en esta institución. Por un lado, se trata de una imagen de "igualdad", ya que todos comen lo mismo y en las mismas condiciones (propio del reconocimiento a la "igualdad de derechos" del enfoque ciudadano $y$ del tipo de autoridad que se pretende ejercer, basada en relaciones más simétricas). Se da entonces el espacio para la charla $y$ el intercambio de anécdotas, los chicos se acercan a los educadores, los abrazan, se les tiran encima, les preguntan cosas, toman la iniciativa del contacto, se intercambian bromas, les cuentan las actividades que hicieron en el centro de día, las que piensan hacer cuando se vayan, comportándose de la manera en que se espera se comporten en tanto niños, en una clima ameno y familiar. Es esta la imagen que la institución intenta mantener sobre sí misma respecto de la interacción con los chicos. Pero, al mismo tiempo, la presencia adulta en las mesas 
garantiza el mantenimiento de cierto control, el seguimiento de las normas y la posibilidad de estar cerca para actuar en el mismo momento en que se genere algún conflicto, lo que da cuenta de una especie de "estado de alerta permanente". Esta tensión latente se expresa, por ejemplo, en la distribución de los cubiertos: cada chico tiene un tenedor o cuchara para comer, pero solo existe un cuchillo en cada mesa, controlado por el educador, para evitar que este elemento sea usado como arma tanto dentro de la institución en caso de estallido repentino de una pelea, como en el exterior. Todos (el educador también) comparten el mismo cuchillo y es el adulto quien permite el uso por los chicos, pero siempre bajo su control.

Los chicos expresan sutilmente desacuerdos y desafían esta situación de control: la más común implica, por ejemplo, que entablen entre sí una conversación en términos propios de la jerga callejera, que haga que el adulto allí presente no pueda participar y que generalmente, incluye alguna referencia despectiva (lo que supone una velada amenaza de cambio de criterio válido en la relación por el del uso de la fuerza física, en la que el adulto ocupa un lugar subordinado); otras veces (y esto especialmente sucede de los chicos varones hacia las educadoras mujeres) con el establecimiento de una conversación plena de ambiguas ( $y$ no tanto) insinuaciones sexuales dirigidas especialmente a la figura de la educadora; también, haciendo "bromas" a los adultos, del estilo de quitarles el llavero que permite el acceso a la institución, o esconder el cuchillo (lo que implica la posibilidad de usarlo como arma, también contra el educador). Otras veces pueden realizar comentarios respecto de actividades ilegales o que implican un peligro que están realizando, que realizaron o que piensan realizar (drogarse, robar, participar de una pelea, realizar juegos que impliquen altos riesgos como saltar de trenes en movimiento, etc.), como una manera de mostrarse peligrosos, lo que suele generar la reacción de los educadores, ya sea en la forma de reprobación explícita ("no hagas eso") o a modo de "consejo".

En general, estas prácticas de desafío se mantienen en un tono ambiguo que pueden en cualquier momento salvarse en términos de "broma". En cambio, si el tono se vuelve violento en el trato con los educadores, o si se establece entre ellos algún tipo de pelea explícita, esta situación lleva rápidamente a la intervención de los demás educadores presentes para intentar suspenderla a través de advertencias, y si persiste, sacándolo directamente del centro de día.

En la situación del almuerzo, puede observarse cómo se realizan distintas operaciones que dan lugar a tal construcción: las operaciones de la diferenciación entre lo propio de los adultos y de los niños (Ariès, 1988), que promueven o sancionan determinadas prácticas, relación con los objetos, "formas de ser" y comportamientos, como legítimos e ilegítimos $y$ el establecimiento de una relación de poder (jerárquica y desigual) entre el estatus de adulto $y$ el de niño, jerarquía siempre relativa al tipo de criterio de que se acuerde entre ambas partes como válido para sostener la interacción y que por lo tanto, mantiene latente la posibilidad de cambiar de criterio y así, de posición jerárquica. Se ve también, que en el caso de que esto no se logre, se produce un límite en la asistencia propuesta y los chicos deben "salir" del centro de día, confirmando las jerarquías de poder de la relación.

Un tercer elemento propio de esta construcción social de la infancia tiene que ver, como se explicó anteriormente, con concebirla como una etapa de transición, que implica el de dejar de ser niño y la transformación en adulto. Este elemento también aparece caracterizando la asistencia que brinda el centro de día y también, como su límite: si la niñez implica la transformación futura en adulto, entonces desde la institución se espera intervenir en tal transformación, que en el caso particular de los 
niños y jóvenes que allí asisten se relaciona con la posibilidad de salir de la situación de calle. En este sentido, importa menos la posibilidad concreta de facilitar esta realidad futura, que la manifestación por parte de estos chicos de un "deseo" de que ese cambio se produzca, ya que ello da muestras de su carácter de "transformables" y justifica la intervención de la institución. Sin esta idea de transformación futura (aunque sea como manifestación de voluntad o "deseo"), la condición de niño (y entonces, la pertinencia de ser asistido) se pone en cuestión. Como aparece explicitado en las palabras de este educador:

En general, los que son chorros $^{21}$, chorros de mano armada, son tipos que... no son los tipos de los problemas simple del chico, ¿entendés? Aunque sea joven, pero tiene armada su vida más de chorro... Te quiero decir: tiene un lenguaje más adulto con vos. (...) [contando el caso de un chico:] ¡Tiene un itinerario de cárcel! (...) ¡Con ése no se pudo hacer nada! ${ }^{22}$.

Aquí, aparece claramente la idea de que la institución puede operar solo sobre aquellos que, en tanto niños, son seres aún indefinidos, no tienen aún su vida "armada". En caso de que sí la tenga, aparece la idea de adulto, independientemente de la edad que tenga el niño o joven. Por lo tanto, la institución no puede operar la "restitución de la niñez" que comprende como objetivo.

\section{PALABRAS FINALES}

El centro de día, en sus prácticas cotidianas, redefine su misión de "inclusión social" como inclusión a la niñez hegemónica. Para los asistentes, llegar a ejercer ese "rol de niño" propuesto (y que implica también la posibilidad de ejercer el rol de "adulto protector y comprensivo que les permita ser niños" por parte

21 Expresión del argot popular argentino que remite a la figura del "ladrón".

22 Entrevista a un coordinador del programa. Fecha: 27-01-2005. de la institución) aparece entonces, como una condición tan importante como la edad, a la hora de definir a quiénes asistir y a quiénes no. Por lo tanto, quienes tengan entre 8 y 18 años (definición formal de "niño"), pero no puedan adecuarse al ejercicio del rol, se verán con el riesgo de quedar por fuera de la asistencia brindada; al mismo tiempo, quienes tengan más de 18 años pero sigan desarrollando y ejerciendo el rol propuesto, podrán en términos generales seguir yendo, por un tiempo, más allá de la edad estipulada. De esta manera, el "ser niño" pasaría de constituir una simple definición de la población destinataria del dispositivo asistencial, a convertirse en una contrapartida moral exigida para ser merecedor de la asistencia y como tal, discutida, aceptada, rechazada y/o negociada, en las interacciones cotidianas, por los trabajadores $y$ por los propios niños y jóvenes.

Sin embargo, la fuerte naturalización de la idea de infancia lleva a que desde las instituciones que trabajan con estas poblaciones, no se perciba esta condición moral como exigencia realizada a cambio de la asistencia. Por lo tanto, no se perciben tampoco los límites de una propuesta tal de asistencia. Si la asistencia termina siendo concebida más en términos de una "conversión moral" que como el ofrecimiento de sostenes materiales e institucionales, estables en el tiempo, que hagan posible el "cambio" buscado, se corre el riesgo de responsabilizar a las personas (porque "no quieren" o "no aprovechan las oportunidades que se les brinda") de una limitación de la propia política social llevada adelante para asistirlos.

\section{BIBLIOGRAFÍA}

Ariès, P. El niño y la vida familiar en el antiguo régimen. Madrid: Taurus (edición en castellano), 1973.

Beaud, S. y Weber, F. Guide de l'enquete de terrain. Produire et analyser des données ethnographiques. París: La Découverte, 1997.

Cohn, C. Antropologia da criança. Zahar, Jorge (ed.). Río de Janeiro, 2005.

Fioravanti, R. H. "Infância e voluntariado no Hospital Pequeno Príncipe”. [Ponencia 
presentada em $25^{\text {a }}$ Reunião Brasileira de Antropologia]. Goiânia-GO, junio 2006.

Fonseca, C. Caminos de adopción. Buenos Aires: Eudeba, 1998.

Frajtag Sauma, J. "Encontros cartografados: reflexões sobre encontros entre meninos e educadores de rua". Cadernos de campo 14/15. Sao Paulo. 2006: 41-63.

Gentile, M. F. "En el CAINA te habla la boca. La interacción cotidiana en un centro de atención para niños $y$ adolescentes en situación de calle, desde la experiencia de los chicos que la frecuentan". Chaves, M. $y$ otros (coords.). Estudios en Juventudes en Argentina I. Hacia un estado del arte 2007. La Plata: RENIJA/ EdULP, 2009.

Gentile, M. F. "Ser niña o niño y 'estar' en la calle. Género y sociabilidad". Julieta Pojomovsky (dir.). Cruzar la calle. Tomo 2. Buenos Aires: Ed. Espacio, 2008.

Gentile, M. F. "L'enfance à la rue. L'expérience de la vie dans les rues chez les enfants d'une institution d'assistance à Buenos Aires". [Tesis para la aprobación del Máster en Sociologie]. Centre d'études des mouvements sociaux (CEMS) EHESSParís, 2006.

Goffman, E. (1959). La presentación de la persona en la vida cotidiana. Buenos Aires: Amorrortu (edición en castellano), 1997.

Goffman, E. Internados. Buenos Aires: Amorrortu, 1972.

Litichever, C. "Trayectoria institucional y ciudadanía de chicos y chicas en situación de calle". [Tesis para la obtención del título de Magister en Diseño y Gestión de Programas y Políticas Sociales]. Buenos Aires: FLACSO, 2009 .
Macri, M. y otros. El trabajo infantil no es juego. Estudios e investigaciones sobre trabajo infanto-adolescente en Argentina (1900-2003). Buenos Aires: Ed. Stella-La Crujía, 2005.

Marshall, T. H. y Bottomore, T. Ciudadanía y clase social. Madrid: Alianza, 1998.

Mauger, G. "Précarisation et nouvelles formes d'encadrement des classes populaires". Actes de la recherche en sciences sociales 136-137. Marzo 2001: 3-4.

Parizot, I. Soigner les exclus. París: PUF, 2003.

Pichon, P. "La construction des publics". Jaques Ion (dir.). Le travail social en débat(s). París: La Découverte, 2005.

Pojomovsky, J. Cruzar la calle. Tomo 1. Cillis, N. y Gentile, M. F. (colaboradores). Buenos Aires: Ed. Espacio, 2008a.

Pojomovsky, J. Cruzar la calle. Tomo 2. Cillis, N. y Gentile, M.F. (colaboradores). Buenos Aires: Ed. Espacio, 2008b.

Simmel, G. Les pauvres. París: PUf (3era. edición en francés), 1995.

\section{DOCUMENTOS / FUENTES}

Centro de día. Revista chicos de la calle. Buenos Aires: Centro de día-GCBA, 1998.

Centro de día. Proyecto institucional. Buenos Aires: GCABA, 2003.

Centro de día. Canción para las fiestas de fin de año. Buenos Aires: CGBA, 2004.

CIDN. Convención Internacional sobre los Derechos del Niño. Nueva York: ONU, 1989.

Fecha de ingreso: $11 / 10 / 2010$

Fecha de aprobación: 07/03/2011 